\title{
Hierarchical Path Metric in Multi-Hop Wireless Networks
}

\author{
Yuanzhu Peter Chen \\ Department of Computer Science \\ Memorial University of Newfoundland \\ St. John's, NL Canada \\ Email: yzchen@cs.mun.ca
}

\author{
Lijun Qian, Dhadesugoor R. Vaman \\ ARO Center for Battlefield Communications Research \\ Prairie View A\&M University, Texas A\&M University System \\ Prairie View, Texas 77446, USA \\ E-mail: lijunqian@ieee.org,drvaman@pvamu.edu
}

\begin{abstract}
In a large mobile ad hoc network, nodes may be organized by a hierarchical address system that reflects the organizational structure of the users within the network. This is typical for many mission-critical applications such as military networks. For such applications, it is important to keep crossorganization data transfer at the lowest possible level for minimal disruption of the traffic within each organization and for security reasons. To measure the degree that a multi-hop path conforms to this "level constraint", we propose a new link metric to quantify the "distance" between two neighboring nodes in terms of their hierarchical addresses. Using this metric, we discover that existing routing protocols that are not aware of the level preference in routing tend to find routes that violate the level constraint frequently. On the other hand, a direct adoption of the distance metric in existing routing protocols produces significant route detours. To deal with this situation, we need to design a new routing protocol to balance the level preference and the topological shortness of routes.
\end{abstract}

\section{INTRODUCTION}

Mission Critical Networks (MCNs) are under intensive research recently due to its wide-spread applications such as in military operations, disaster relief, etc. Usually an MCN requires fast deployment as well as elimination of infrastructure support. This makes the wireless ad hoc networking technology a promising candidate for MCNs. In an MCN, multiple organizations are typically involved and they are deployed to the same geographical area, each focusing on a potentially different task. For example, police, national guard, and medical teams were deployed to New Orleans after Hurricane Katrina. Another example is the battlefield, where many troops, UAVs, and wireless sensor networks are deployed. It is also typical that each organization has its own communication network within the common geographical area. One unique challenge, as well as opportunity, in an MCN is that multiple organizations need to collaborate, and yet such collaboration should introduce minimum disruption to each other's operation.

In this work, we focus on the wireless communication aspects of collaboration and we assume that the wireless ad hoc networks of different organizations are inter-operable. Specifically, we focus on the routing problem in scenarios where nodes that belong to one organization may relay traffic for another organization. This can happen for various reasons. For instance, at times a node may be out of the radio range of its own network. An illustrative example is provided in Fig. 1, where the oval nodes and the square nodes belong to two different organizations and they are inter-operable. Assume that node $H$ is out of range of any of its peers in its own network. Node $H$ can only communicate with node $A$ through node 1 which belongs to a different organization. In many cases, a helping hand from another organization may save a lot of resources that could be critical for the one being helped. Again, as in the figure, suppose that the oval network is resource constrained. Now if node $A$ wants to communicate with node $G$, a shortcut (4-hop path: $A K 2 J G$ ) may be taken, with the help of node 2, instead of a long route (6-hop path: $A B C D E F G$ ) within the oval organization.

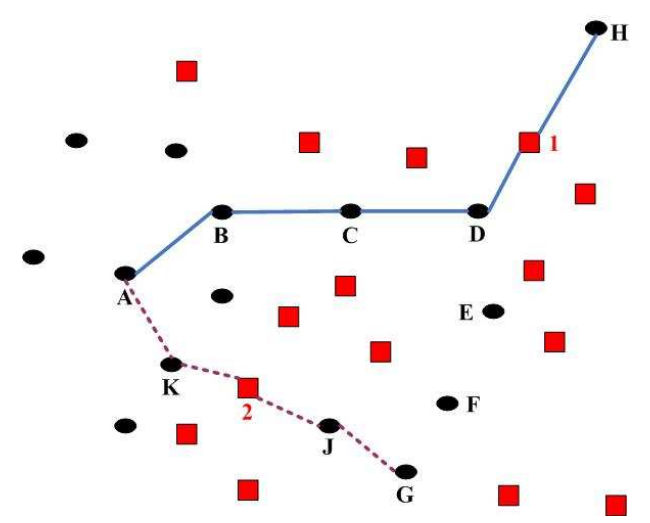

Fig. 1. Nodes of two co-located organizations

Although there are considerable benefits for different organizations to collaborate, we ought to be cautious that the incurred additional overhead to the helping organization should not disrupt its own operation. We are particularly interested in a new routing metric to reflect this unique requirement. In addition, there can be a hierarchy of multiple sub-groups within each organization. Formally, the mixture of multiple organizations, along with the sub-groups therein, can be regarded as one hierarchical network, where it contains a number of tiers of groups. Hence, in this paper, we study the organizational structure of the network by considering hierarchical addresses of the nodes. Specifically, we propose a simple method to represent the "level" of each node by 
its organization, sub-group, etc. Then the routing decision in such networks should take into account the "level constraint" such that cross-organization traffic flow will remain at the lowest possible level. For this purpose, we also propose a metric to measure the "organizational distance" between nodes and evaluate it through simulation. It is demonstrated that the existing popular wireless ad hoc network routing algorithms violate the level constraint frequently in many situations. Our discoveries provide grounds for further investigation of novel routing protocols to balance the level preference and the topological lengths of paths in MCNs.

There are a number studies of inter-domain routing for both wired and wireless networks, such as BGP [1] and HLP [2] for the Internet and those in [3] for wireless ad hoc networks, just to name a few. However, those studies consider a fairly different scenario where different networks are also in different geographical/logical areas and there is pre-defined interface between different these networks. In other words, usually each network is an autonomous system (AS) and nodes in different ASs will not mix and communicate directly. Furthermore, the traffic flows are facilitated intentionally to cross multiple ASs rather than the above-stated reasons for MCNs. In addition, these studies focus on such issues as routing convergence, scalability and QoS of inter-domain routing. Although the organization ID of the nodes is considered in [3], again, the ad hoc networks are located in different geographical areas and the traffic flows are meant to cross different networks. Furthermore, the level constraint is not considered even when there are only two tiers in the system.

The rest of this paper is organized as follows. In Section II, the system model and the problem formulation are given. A method to quantify the degree that nodes of different organizational units are mixed with each other is formulated in Section III. Section IV is dedicated to simulative studies and our findings out of these experiments. Section V concludes the paper and points out some future extension of this paper.

\section{ORGANIZATION-AWARE LINK METRIC}

Assume we have a communication network with a builtin hierarchical address system such that data flows should traverse the lowest units possible to get from the source to its destination. This is typical for a network with strong organizational constraints such as military troops. When no overlapping is allowed (i.e. each node belongs to exactly one unit in the hierarchy), such an addressing system can be organized as a forest-like structure. Here, we formulate an organization-aware link metric for such a type of networks.

\section{A. Network with hierarchical address system}

We denote the set of nodes in the network by $V$. For each node $v$ in $V$, it has a hierarchical address of $h$ levels, denoted by $\left\langle v_{1}, v_{2}, \ldots, v_{h}\right\rangle$, where each component is taken from a countable set, such as an octet of the IP address. As a convention, we use the right most address component for the deepest (lowest) level in the hierarchy. We assume that the addresses are unique in the network. Given two nodes

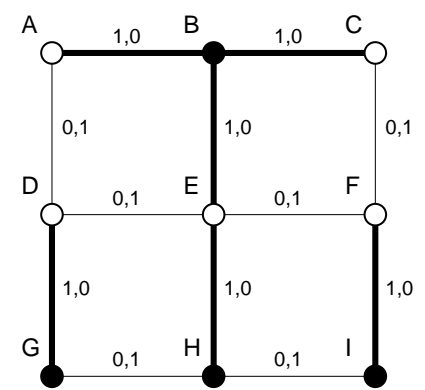

Fig. 2. Paths in a 2-level network

$u=\left\langle u_{1}, u_{2}, \ldots, u_{h}\right\rangle$ and $v=\left\langle v_{1}, v_{2}, \ldots, v_{h}\right\rangle$, we define the bond of them, $b(u, v)$ or simply $b$ when there is no confusion, as the maximum index $b$ in their addresses such that $u_{i}=v_{i}$, for $i=1,2, \ldots, b$, but $u_{b+1} \neq v_{b+1}$. That is, nodes $u$ and $v$ belong to the same unit as deep as level $b(u, v)$. Specifically, when $b=0$, these nodes belong to two different units at the highest level; when $b=h-1$, they belong to the same lowestlevel unit. For example, the network in Fig. 2 has 9 nodes which belong to two different units, indicated by their colors. The bond of nodes $A$ and $D$ is 1 while it is 0 for $A$ and $B$.

\section{B. Organization-aware paths}

In a network of nodes with hierarchical addresses defined as above, it is usually required that traffic flows are kept "as low as possible" in terms of the organizational unit. Formally, we can use an undirected graph $G=(V, E)$ to represent the network, where $V$ is the set of nodes and $E$ is the set of communication links between neighboring nodes. Given a simple path $P=e^{1} e^{2}, \ldots, e^{l}$ between source $s$ and destination $t$ in $G$, we define the bond of a path as

$$
b(P)=\min _{i=1}^{l}\left\{b(u, v) \mid e^{i}=(u, v)\right\} .
$$

That is, the bond of $P$ is the highest level of common unit that it traverses. For instance, path $A D G$ in Fig. 2 has a bond of 0 while path $A D E$ has 1 . For a pair of source and destination, $s$ and $t$, we would want to identify paths connecting them with the maximum bond. In addition, among these paths, we should use the shortest one(s) for data transportation. The shortest path defined in this sense is called an organizationally shortest path (OSP for short).

Following notes should be taken when studying organization-based routing. First, a link metric is necessary for calculating the length of a path, which should also reflect the "level" of the link, i.e. the bond of its endpoints. Here, such a metric can be based on any traditional link weight notion. For example, a unit link weight can be employed so that the path length is essentially the hop count. Alternatively, we can also use more informative metrics such as the Expected Transmission Count (ETX) [4] and the Expected Transmission Time (ETT) [5]. Second, the identification of an OSP should permit different strategies, such as pro-active table driven and reactive on-demand. Consequently, existing 
model and IEEE 802.11 MAC settings in ns-2, which translates to a transmission range of 250 meters in a 2-dimensional space. We employ three node deployment scenarios of the same approximate node density as in Table I. In each scenario, a network is associated with a hierarchical address system of $h(h=2,3,4$ here) layers. All nodes have unique addresses in the system. We have two degrees of mixing of the addresses in each scenario:

TABLE I

SCENARIO PARAMETERS

\begin{tabular}{c|c|c|c}
\hline Area & \# nodes $(n)$ & \# layers $(h)$ & Notation \\
\hline \hline $3000 \times 3000 \mathrm{~m}^{2}$ & 400 & 4 & $s_{1}$ \\
\hline $1500 \times 1500 \mathrm{~m}^{2}$ & 100 & 3 & $s_{2}$ \\
\hline $750 \times 750 \mathrm{~m}^{2}$ & 25 & 2 & $s_{3}$ \\
\hline
\end{tabular}

- Uniform distribution $\left(d_{1}\right)$ - For each node, all components of the address vector follow a uniform distribution in the address space. In this case, the nodes of different organizations are very well mixed amongst each other.

- Conglomerate distribution $\left(d_{2}\right)$ - Nodes of the same lowest-level organization are close to each other geographically. Thus, the nodes of different organizations are barely mixed with each other.
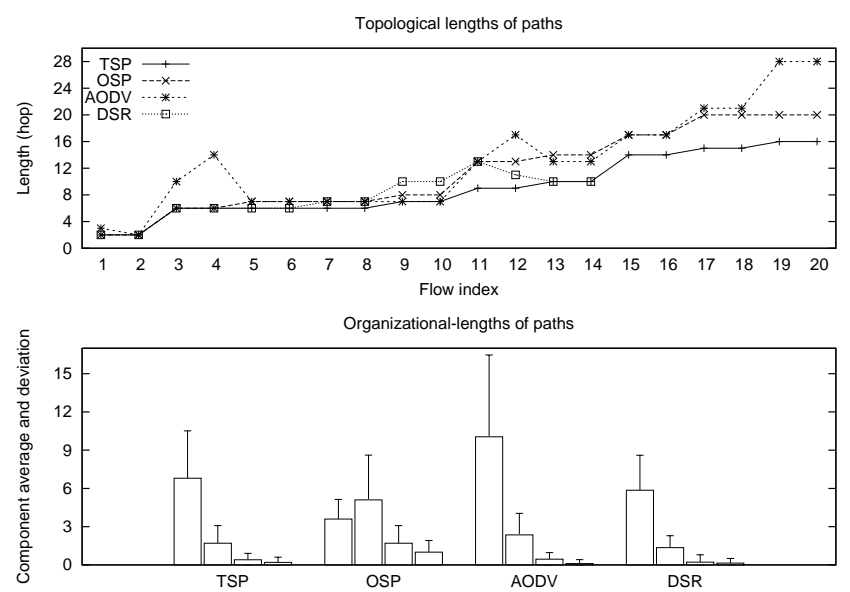

Fig. 4. Topological and organizational path length comparison - $\left(s_{1}, d_{1}\right)$

We use AODV and DSR as benchmark to measure paths used in a network. These widely used and well understood routing protocols stabilize in a static multi-hop wireless network. To trace the routes adopted by the routing protocol, we assign 10 pairs of bi-directional CBR flows between 10 randomly selected node pairs. These CBR flows are lightweight at the rate of 1 packet per second and of 500-byte lengths. They are run in each of the six scenario-distribution combinations. In the meantime, we export the network structure from the simulator and use a separate program to calculate the TSPs and OSPs between the corresponding node pairs. These globally calculated paths are used as references for the routing protocols.
TABLE II

MIXES OF SCENARIOS

\begin{tabular}{c|c|c}
\hline & $d_{1}$ & $d_{2}$ \\
\hline$s_{1}$ & $\langle 5.81,1.55,0.345,0.17\rangle$ & $\langle 0.51,1.185,1.795,4.29\rangle$ \\
\hline$s_{1}$ & $\langle 5.22,1.7,0.64\rangle$ & $\langle 1.3,1.48,4.84\rangle$ \\
\hline$s_{1}$ & $\langle 5.12,1.68\rangle$ & $\langle 1.92,3.84\rangle$ \\
\hline
\end{tabular}

We first focus on the large network of 400 nodes with a 4 level address system with uniform node distribution $\left(s_{1} \times d_{1}\right)$. The upper plot of Fig. 4 is the topological lengths of the 20 paths used to transport the 10 CBR flows. Four kinds of paths are investigated here, i.e. the topologically shortest paths (TSP), organizationally shortest paths (OSP), paths used by AODV (AODV), and those used by DSR (DSR). The paths are indexed by the flow IDs. For better readability, we rank these paths according to an increasing TSP length. We can see that the OSP between a node pair is generally about $25 \%$ longer than the corresponding TSP, but they are of about the same lengths as those calculated by AODV and DSR. In our experiments of 50 seconds, we notice that when a pair nodes are more than 14 hops apart, DSR cannot find a connecting path in time, which is indicated by the discontinuation of the last curve after index 15 . In the lower plotting of the figure are the average organizational lengths of these paths and their standard deviations. Each cluster of bars corresponds to one type of paths, and the $i$ th bar in a cluster is the $i$ th level of the vector path metric. We can see that the OSPs have about half of the highest component as those of TSP. Note that for random flows across a large network, it can be inevitable that some flows must go between different highestlevel organizations. OSP in this case can, however, halve such occurrences. The organization-unaware AODV and DSR protocols have a similar organizational metric as TSP although somewhat larger topological lengths. (The values for DSR are under-estimated due to its failure in finding long paths as stated previously.)
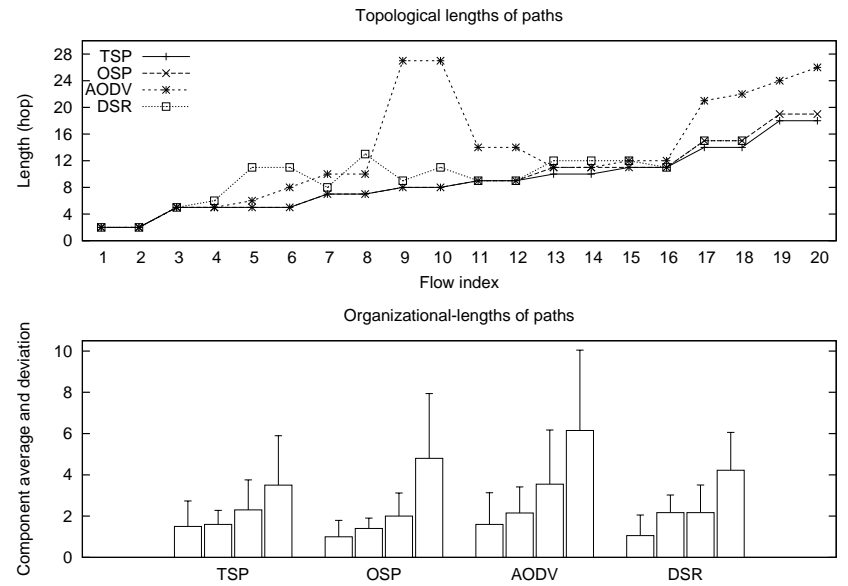

Fig. 5. Topological and organizational path length comparison $-\left(s_{1}, d_{2}\right)$ 
Next, we study the same large network scenario but with conglomerate node distribution $\left(s_{1} \times d_{2}\right)$. In this case, the "mix" of the network is $\langle 0.51,1.185,1.795,4.29\rangle$ (Table II), where it is $\langle 5.81,1.55,0.345,0.17\rangle$ for the previous case. Such a difference in the mix values indicate that their degree of mixing of nodes are significantly different (Section III). Same measurements are presented as the previous case. We observe that when the nodes of organizations are extremely conglomerate, a TSP tends to be an OSP in many cases. In terms of measurement, the topological lengths of these two types of paths are almost identical (upper chart); yet the OSPs can still reduce the value of the first component in the organizational metric to about $70 \%$ whenever possible as indicated by our experiments (lower chart).
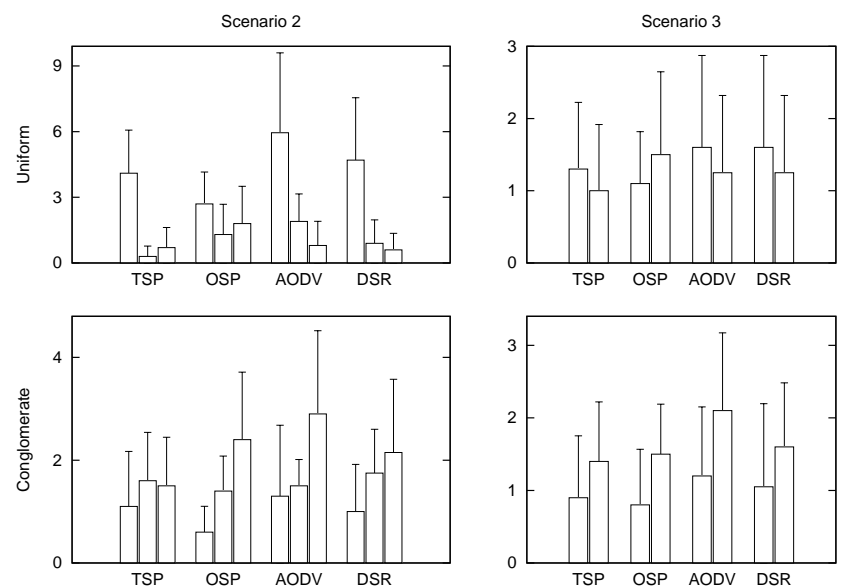

Fig. 6. Organizational path length comparison $-\left(s_{2,3}, d_{1,2}\right)$

We further summarize our experimental measurements for the combinations $s_{2} \times d_{1}, s_{2} \times d_{2}, s_{3} \times d_{1}$, and $s_{3} \times d_{2}$ in Fig. 6. The mixes of these scenario-distribution combinations are in Table II. Again, we observe that OSPs are particularly effective in keeping traffic as low as possible for high-mixing distribution.

\section{CONCLUSION AND FUTURE RESEARCH}

In a multi-hop wireless network, nodes can be associated with addresses that reflect the organizational structure amongst themselves. Due to the wireless and potentially mobile nature of these nodes, nodes belonging to the same unit in the organization can be scattered around the entire network. Data transfer in such a network sometimes is required to traverse units as low-level as possible for minimal disruption and maximal privacy reasons. In this work, we devise a vector path metric that takes the organizational structure of the network into account. To test the effectiveness of this metric using a packet simulator, we compare routes adopted by some routing protocols in ad hoc networking to those calculated globally and study their relative topological and organizational lengths. Our findings indicate that this metric captures the organizational notion in path length very well. In addition, in various network sizes and node distributions, an OSP usually has a considerably smaller organizational length than the TSP connecting the same pair of nodes, while its topological length is merely slightly greater than that of the TSP. As part of the future research, both the metric itself and routing protocol design to utilize this metric are worth investigation.

The organizational path metric is essentially a generalization of previous link/path metrics in the literature. The lexicographical order used to compare this metric can be regarded as an extreme case of radix-based comparison, where the radix in this case is infinity. When the radix is 1 , the comparison and path calculation are essentially the conventional nonorganizational metric. In general, a finite value of the radix can be used to strike the balance of how paramount the "aslow-as-possible" data transfer requirement is.

The globally computed OSPs apparently can only be used as a reference or bound. In a multi-hop wireless network, it is critical that paths with small organizational lengths can be quickly calculated by relevant nodes in a distributed and cooperative fashion. Directly adopting this vector link metric in existing routing algorithms is an option but not necessarily a good one because the settling time of stable route discovery will be long. That is, these algorithms use control packet broadcasting one way or the other and, in most cases, the propagation rate of such control packets do not carry the organizational notion but only reflects the hop length and link throughput [4], [5]. As a result, it will take a much longer time for a routing protocol thus designed to find a short (or shortest to be optimistic) path in the organizational sense. Therefore, an organization-aware routing protocol is imperative as an immediate conclusion of this work.

\section{REFERENCES}

[1] Y. Rekhter and T. Li, "A border gateway protocol 4 (BGP-4)," RFC 1771, March 1995.

[2] L. Subramanian, M. Caesar, C. T. Ee, M. Handley, M. Mao, S. Shenker, and I. Stoic, "HLP: A next generation interdomain routing protocol," in Proceedings of ACM SIGCOMM, August 2005.

[3] W. Ma and M. C. Chuah, "Comparisons of inter-domain routing schemes for heterogeneous ad hoc networks," in IEEE International Symposium on a World of Wireless Mobile and Multimedia Networks (WoWMoM), Taormina, Italy, June 2005, pp. 368-373.

[4] D. S. J. D. Couto, D. Aguayo, J. Bicket, and R. Morris, "A highthroughput path metric for multi-hop wireless routing," in Proceedings of MobiCom, San Diego, CA, September 2003, pp. 134-146.

[5] R. Draves, J. Padhye, and B. Zill, "Routing in multi-radio, multi-hop wireless mesh networks," in Proceedings of MobiCom, Philadelphia, PA, September 2004, pp. 114-128.

[6] C. E. Perkins and E. M. Royer, "Ad-hoc on-demand distance vector routing," RFC 3561, July 2003.

[7] D. Johnson, Y. Hu, and D. Maltz, "On the dynamic source routing protocol (DSR) for mobile ad hoc networks for IPv4," $R F C 4728$, February 2007. 